\title{
Tasa metabólica basal ¿una medición sin fundamento adecuado?
}

\author{
Basal metabolic rate, a medition without adequate foundation? Review \\ article
}

\author{
Sánchez $P E^{1}$, Polanco JP2, Rosero $R J^{1 *}$
}

${ }^{1}$ Médico Internista y Endocrinólogo, Centro de Obesidad

Dismetabolismo y Deporte (COD2), Clínica Las Américas, Medellín, Colombia.

${ }^{2}$ Médico Internista y Epidemiólogo, Centro de Obesidad

Dismetabolismo y Deporte (COD2), Clínica Las Américas, Medellín, Colombia.

\section{Autor de correspondencia:}

Pedro Enrique Sánchez, Ricardo J. Rosero Revelo

\section{Correo electrónico:}

pedros0822@hotmail.com, cod2@clincalasamericas.com.co

Fecha de recepción: 25/06/2019

Fecha de aceptación: 30/10/2019

\section{Resumen}

La energía necesaria para la regulación de las funciones fisiológicas depende del equilibrio entre el aporte y el gasto energético. Un disbalance entre estas condiciones, donde se vea favorecido el consumo sobre el gasto, lleva a un incremento de la reserva, el cual, a su vez, favorece la hipertrofia e hiperplasia del tejido adiposo; en condiciones crónicas, este reservorio energético lleva a la obesidad. A través del uso de fórmulas, se ha tratado calcular la tasa metabólica basal para considerar el aporte óptimo energético y, por ende, la individualización en la prescripción de estrategias nutricionales en el paciente sano y con enfermedades crónicas no transmisibles (ECNT). Sin embargo, estas fórmulas no han sido evaluadas ni validadas para todas las poblaciones y menos en obesidad, que es, de forma coincidente, la población donde más se usan en la práctica clínica, además del poco conocimiento en medicina y la mayor aplicación por nutrición. El propósito de este artículo es revisar los elementos que constituyen las fórmulas de cálculo de la tasa metabólica basal (TMB) y la población de estudio para evidenciar la usabilidad de estas fórmulas en la práctica clínica en pacientes sanos y con ECNT, como la obesidad y diabetes mellitus, dada la importancia que esto representa en el contexto del balance energético.
Palabras clave: metabolismo basal, metabolismo energético, obesidad, hipertensión, diabetes mellitus.

\begin{abstract}
The energy necessary for the regulation of physiological functions depends on the balance between the contribution and energy expenditure. An imbalance between these conditions, where consumption is favored over expenditure, leads to increased reserve, which in turn favors hyperplasia and hyperplasia of adipose tissue, as a form of energy reservoir. In view of the above, an attempt has been made to continuously search for the use of formulas for the calculation of the basal metabolic rate for the consideration of energy intake and, therefore, the prescription of food. However, these formulations have not always been evaluated or validated for all populations and less in obesity, which coincidentally is the population where they are most used in clinical practice, and in addition to little knowledge in medicine and greater application by nutrition. The purpose of the article is to review the elements that make up the TMB calculation forum and the study population to demonstrate the usability of these formulas in clinical practice.
\end{abstract}

Keywords: Basal metabolism, energy metabolism, obesity, hypertension, diabetes mellitus.

\section{Introducción}

El sobrepeso y la obesidad son la nueva pandemia del siglo XXI. Sus cifras se han duplicado en las últimas 3 décadas, llegando a haber más de 1,9 billones de casos de sobrepeso y 609 millones de obesidad en mayores de 18 años (1). En la actualidad, es un problema social y se ha convertido en un reto para la salud pública al ser un factor de riesgo para condiciones como la hipertensión, dislipidemia, diabetes, eventos cerebro y cardiovasculares, problemas osteomusculares y varios tipos de cáncer (2-7). Para enfrentar esta problemática se han propuesto programas de educación basados en estilos de vida saludable, que incluyen, como piedra angular, el consumo ba- 
lanceado de nutrientes y el ejercicio, buscando así mantener un balance energético entre el consumo y el gasto (8). Dado que todos los sistemas biológicos se rigen por las leyes de la termodinámica, acorde a lo cual "la energía no se crea ni se destruye, solo se transforma”, en un sistema viviente, como el ser humano, la energía química proveniente de los alimentos puede transformarse en calor, energía eléctrica, mecánica o en energía en reposo; la energía utilizada en condiciones basales se denomina gasto energético, y depende de cuatro factores: la tasa metabólica basal (TMB), el efecto termogénico de la comida, la actividad física y el cambio en la temperatura del ambiente (9).

La TMB es la energía indispensable para el desarrollo y mantenimiento de las funciones orgánicas básicas (10). Corresponde aproximadamente al 50 \%-75 \% del gasto energético total (GET) y se relaciona con aquella energía utilizada cuando el cuerpo está en reposo físico y psicológico, luego de una noche de sueño, sin ninguna actividad física previa, posingesta de al menos 10 horas y en un ambiente neutro; por otra parte, la tasa metabólica en reposo (TMR) solo necesita 2-4 horas de ayuno y es $10 \%$ de la TMB. (9). Esta última suele permanecer estable por largos períodos, sin embargo, es susceptible de cambiar y puede utilizarse para el seguimiento de los pacientes mediante métodos, como la calorimetría, a pesar de la alta variabilidad interindividual según la composición corporal, edad, sexo y etnia (11). El resto del GET es la cantidad de energía gastada para la actividad física (20\%-40\%), el efecto térmico de las comidas (5\%-30 \%) y, en algunos casos, las respuestas adaptativas a termogénesis y estrés psicológico (12).

En la práctica clínica, a la TMB se le ha dado un enfoque erróneo al establecer patrones que, sin evidencia científica fuerte, buscan explicar la facilidad o no de ganar peso, denominados metabolismo rápido o lento, los cuales han sido el fundamento de muchos productos en el mercado que los denominan aceleradores metabólicos. Por esta razón, es importante recordar este parámetro de medición, los componentes principales para su cálculo, comprender cuándo y en quién utilizar las fórmulas predictivas de TMB para obtener una adecuada confiabilidad, dejando siempre la pregunta si este cálculo es factible y confiable en la población con obesidad con o sin comorbilidades, la cual se promueve en diferentes áreas clínicas y, por su mal entendimiento, podría conllevar a una mala práctica en dicha población.

\section{Métodos}

Se realiző una revisión bibliográfica internacional de los artículos publicados en las bases de datos PubMed, EBSCO host, LILACS, Medline, OVID y CLINICAL KEY, sin aplicación de límite temporal, ya que uno de los objetivos de la revisión fue buscar el origen y la utilidad de las diferentes ecuaciones predictivas de TMB en distintas poblaciones. La selección de los artículos se realizó en función de criterios de estudios con metodología cuantitativa y cualitativa, revisiones bibliográficas, opinión de expertos, artículos originales y estudios aleatorizados realizados tanto en humanos como en animales en idioma inglés y español; además, se incluyeron los términos DeCS: "metabolismo basal", "metabolismo energético", "obesidad", "hipertensión", "diabetes mellitus". Luego se hizo un análisis crítico sobre el tema, avalado por la información encontrada en la literatura consultada.

\section{Medición de tasa metabólica}

La TMB se expresa por unidad de medida del índice metabólico (MET), el cual equivale aproximadamente a $1 \mathrm{kcal} / \mathrm{kg} / \mathrm{h}$, lo que corresponde a $4184 \mathrm{~kJ} / \mathrm{kg} / \mathrm{h}$ (13). En la práctica clínica y de laboratorio se ha utilizado tradicionalmente métodos no invasivos, como la calorimetría indirecta (CI), con técnicas de medición basados en circuitos abiertos y cerrados, donde se mide el consumo de $\mathrm{O}_{2}$ y producción de $\mathrm{CO}_{2}$ bajo condiciones de termoneutralidad, reposo físico y mental, estándares de presión atmosférica, ayuno y ausencia de otros factores, como tabaquismo, medicamentos, fármacos psicoactivos y trastornos tiroideos no controlados.

En el circuito cerrado, el $\mathrm{CO}_{2}$ producido es absorbido por el sistema y el $\mathrm{O}_{2}$ se añade para mantener el volumen de gas constante; el consumo de $\mathrm{O}_{2}$ se calcula de la tasa promedio de disminución del volumen desde el espirómetro. En promedio se ha observado que esté método de CI tiende a producir mayores niveles de TMB. Los elementos requeridos para el cálculo por CI no siempre están disponibles, por lo cual, múltiples ecuaciones predictivas se han desarrollado a lo largo de los años para lograr su cálculo de forma rápida, sencilla y económica, teniendo en cuenta las características de los individuos (9). La mayoría de estos modelos predictivos incluye parámetros determinados a nivel molecular (masa, grasa), celular (líquidos y sólidos extracelulares), órgano/tisular y corporal total (masa corporal) (14).

Múltiples estudios se han realizado en poblaciones específicas, tomando como estructura los mismos parámetros, sin encontrar una correlación adecuada, dado que estas ecuaciones fueron creadas usando, en su mayoría, hombres y jóvenes blancos, saludables, de peso normal u obesos y puede afectarse por condiciones como el ejercicio durante o previo a su medida, ingesta reciente de comida, cambios de temperatura corporal, talla, peso, área de superficie corporal, género, edad, reproducción, lactación, estado emocional, niveles circulantes de hormona tiroidea, epinefrina y norepinefrina (9). Los órganos internos, como el corazón, cerebro, hígado y riñón, que corresponden al $7 \%$ del peso corporal, son responsables del $60 \%$ de la TMB (15). El músculo que corresponde al $40 \%$ del peso es responsable del $18 \%$, teniendo normalmente el depósito de tejido graso un rol menor, sin embargo, al aumentar su cantidad, como es el caso de los pacientes obesos, esta propor- 
ción puede cambiar (16). Al evaluar el grado de concordancia entre ecuaciones predictivas y los valores de CI, estudios como el de Pavlidou y colaboradores, en población caucásica, no encontró concordancia estadísticamente significativa entre la TMB medida y la estimada por distintas fórmulas, como la de Harris y colaboradores, Roza y colaboradores, Achofield, Owen, FAO/WHO/UNU, Mifflin y colaboradores, Harrington y colaboradores, así como otras descritas a lo largo del tiempo que incluyen la de Quenouille y colaboradores, donde, a pesar de haber una aproximación razonable, se encontró que debe tomarse en cuenta el índice de masa corporal en las ecuaciones de predicción para obtener mejores resultados, pues este tiene una relación inversa con la TMB (10, 17-24). Incluso, debe tenerse en cuenta la localización de residencia, pues se ha observado una sobreestimación de los valores con las formulas predictivas de hasta un $18,9 \%$ en individuos que viven en latitudes tropicales (25-27). Recientemente, con la base de datos Oxford, se evaluó hombres y mujeres, distribuidos en 6 grupos de edad, una nueva ecuación predictiva, y se encontraron menores valores de TMB al compararlo con la FAO/WHO/ UNU en hombres de 18-60 años y mujeres de todas las edades (Tabla 1) (28).

Dada la importancia de la composición corporal en algunas de las ecuaciones predictivas, en el caso de pacientes con obesidad se han realizado estudios, como el de Achamrah, el cual evaluó el impacto de los métodos de composición corporal por densitometria (DXA) o bioimpedanciometría en la exactitud de los resultados; al comparar la CI con las fórmulas predictivas se encontró una exactitud de las ecuaciones en el mejor de los casos en el 68 \% y esta disminuía en pacientes con IMC $>40$ (33).

\section{Tasa metabólica y composición corporal}

Se ha atribuido la variabilidad intra e interindividual de la TMB a las diferencias en la composición corporal. En personas sanas, la masa magra (MM) es responsable del mantenimiento de la fuerza y funcionalidad del individuo; a esta se le atribuye $80 \%-90 \%$ de la TMB total. El tronco es un fuerte predictor de TMB y, a nivel periférico, es un indicador de salud metabólica. A la masa grasa (MG) tradicionalmente se le ha atribuido un menor impacto metabólico, siendo menos del $5 \%$ de la TMB en personas delgadas (34). Sin embargo, al ser el tejido adiposo un órgano de distribución global, con capacidad de expansión en condiciones de exceso energético, este cobra mayor relevancia en la obesidad, dado que, al aumentar la proporción del tejido adiposo blanco a nivel subcutáneo y visceral, conlleva un mayor impacto metabólico de forma directa debido a los cambios que genera en la función mitocondrial (16).

A nivel del substrato oxidativo, y de forma indirecta a través de cambios crónicos, dado el aumento en los niveles de adipoquinas, que han mostrado relación directa con la TMB, entre ellas, la leptina se promueve la oxidación lipídica y la biogénesis mitocondrial y se acelera el gasto energético en los tejidos periféricos; la adiponectina estimula la oxidación lipídica y la respuesta antiinflamatoria, siendo un marcador de trastornos metabólicos; la vaspina y la proteína 4 de unión a retinol (RBP4) (35-39). Sepandar y colaboradores evaluaron la TMB por CI en 263 mujeres con síndrome metabólico e IMC promedio de $33,28 \mathrm{~kg} / \mathrm{m}^{2}$, en el que se encontró la omentina 1, la cual mejora la acción de la insulina, tiene una papel significativo en la regulación de la TMB, en este caso, sin encontrar resultados concluyentes con la RBP4 y la vaspina (40-41).

La grasa visceral es más activa metabólicamente al estar asociada con hiperinsulinemia e hipercortisolismo, siendo inversamente proporcional a la tasa de oxidación grasa y flexibilidad metabólica; por otra parte, la grasa subcutánea se relaciona con mayores niveles de leptina y estradiol, así, como con mayor oxidación grasa (16). Es por esto, entre otras razones, que es importante conocer la distribución corporal como parte del estudio del estado metabólico. Hirsch y colaboradores determinaron la relación de la composición corporal total y por segmentos asociado con la TMB medido por DXA y CI en adultos con sobrepeso y obesidad. Los autores encontraron que la TMB se correlaciona positivamente con la $\mathrm{MG}(\mathrm{R}=$ $0,535, p=0,009)$ y MM ( $\mathrm{R}=0,645, p=0,001)$ en hombres y en mujeres $M G(R=0,784, p<0,001)$ y $M M(R=0,867, p<0,001)$.

Al realizar el análisis por segmentos hubo una relación positiva con MM en brazos ( $R=0,583)$, piernas $(R=0,664)$ y tronco $(\mathrm{R}=0,593)$ y $M G$ en brazos $(\mathrm{R}=0,511)$ y tronco $(\mathrm{R}=$ 0,573 ) en hombres, y para mujeres con todos los segmentos de $M M$ y $M G(R=0,522-0,879)$, siendo más fuerte la MM en tronco $(\mathrm{R}=0,879)$ y piernas $(\mathrm{R}=0,821)(42)$. En otro estudio, Sabounchi y colaboradores encontraron que la masa libre grasa es el mejor predictor independiente de TMB, teniendo la MG un coeficiente de relación positivo moderado, sin embargo, estas dos parecen tener un impacto independiente, lo que explica la mayoría de las variaciones de la TMB (43).

\section{Tasa metabólica como predictor de aumento y reducción de peso}

Se ha descrito que cada kilogramo de masa corporal gasta entre 3 y $14 \mathrm{kcal} /$ día en reposo, lo que supondría que una variación en el peso fuese inversamente proporcional a la TMB, siendo una baja TMB un predictor independiente de ganancia de peso a largo plazo, lo cual ha quedado en evidencia en estudios como el de Pimjai y colaboradores, donde, en una muestra heterogénea de 757 sujetos que incluía sujetos delgados, obesos, adultos y jóvenes metabólicamente normales y resistentes a la insulina, luego de ajustar por composición corporal, edad y género, se encontró que aquellos adultos con baja TMB, correspondiente al $15 \%$ inferior, están más predispuestos a ganar peso que aquellos con alta TMB ubicados en el $15 \%$ superior, así como que la tasa de cambio de peso en kilogramos y porcentaje por año fue numéricamente menor en los indivi- 


\section{मnd \\ healthcare within reach}

\section{X-Insight}

Deja que la tecnología X-Insight te acompañe

\section{Solución perspicaz}
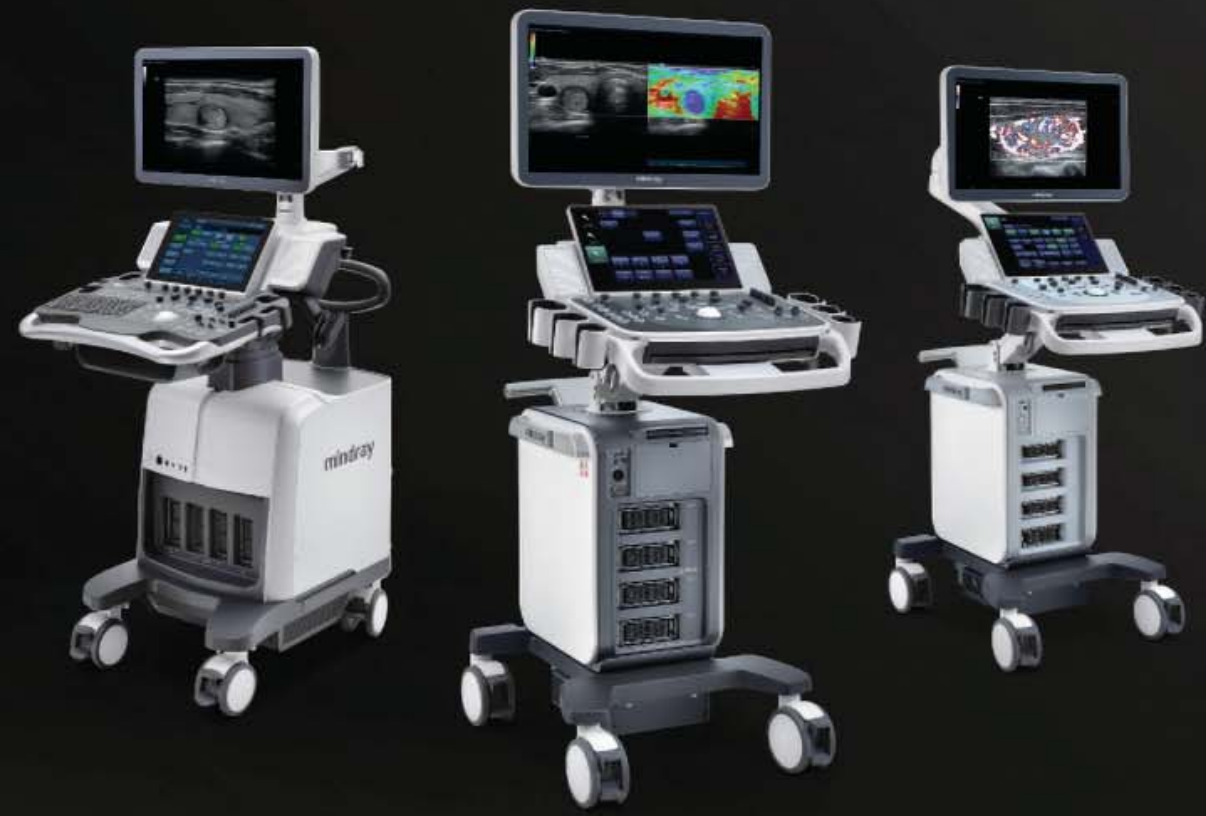

- Más claridad con menos esfuerzo

- Comodidad con alta productividad

- Inteligencia a través de exámenes completos

MINDRAY MEDICAL COLOMBIA S.A.S.

Av. Calle 100 No. 19 - 54, Of. 1002 -

Bogotá, D.C. - Colombia

Tel. (57-1) 3130892 - 3120892

Síguenos en nuestras redes sociales

E-mail: info@mindray.com 


\begin{tabular}{|c|c|c|c|}
\hline Referencia & Población utilizada & $\begin{array}{l}\text { Ecuación para la estimación de TMB } \\
\qquad(\mathrm{kcal} / \mathrm{d})\end{array}$ & $\begin{array}{l}\text { Población } \\
\text { objetivo }\end{array}$ \\
\hline $\begin{array}{l}\text { Harris y Benedict } \\
(1984)(18,29)\end{array}$ & $\begin{array}{l}n=239 \text { sujetos blancos, } 16-63 \\
\text { años: } 136 \text { hombres: peso } 61,1 \pm \\
\text { 10,3 kg con edad de } 27 \pm 9 \text { años; } \\
103 \text { mujeres: peso } 56,5 \pm 11,5 \mathrm{~kg} \\
\text { con edad de } 31 \pm 4 \text { años }\end{array}$ & $\begin{array}{l}\text { Mujer: } 655,0955+(9,5634 \times \mathrm{MC})+(1,8496 \\
\times \mathrm{A})-(4,6756 \times \text { edad }) \\
\text { Hombre: }(13,75 \times \mathrm{MC})+(5,003 \times \text { altura })- \\
(6,755 \times \text { edad })+66,47\end{array}$ & Sana \\
\hline Quenouille (1951) (24) & $\begin{array}{l}\mathrm{N}=8600.4300 \text { de } 17-39 \text { años, } 800 \\
\text { mayores } 40 \text { años y } 3520 \text { menores } \\
\text { de } 1 \text { año. }\end{array}$ & $\begin{array}{l}\text { Mujer: }(2,975 \times A)+(8,90 \times M C)+(11,7 \times \\
\text { SC })+(3,0 \times H)-(4 \times T)+293,8\end{array}$ & $\begin{array}{l}\text { Sanos. Involucra } \\
\text { gente que vive } \\
\text { en el trópico, } \\
\text { evaluando efecto } \\
\text { de etnia y clima }\end{array}$ \\
\hline $\begin{array}{l}\text { FAO/WHO/UNU } \\
(1987)(21)\end{array}$ & $\begin{array}{l}\mathrm{n}=11000 . \text { Varios grupos étnicos y } \\
\text { amplio rango de IMC. }\end{array}$ & $\begin{array}{l}\text { Mujer (18-30): }(14,7 \times M C)+496 \\
\text { Mujer (30-60): }(8,7 \times M C)+829 \\
\text { Mujer (> 60): }(10,5 \times M C)+596 \\
\text { Hombre (18-30): }(15,3 \times M C)+679 \\
\text { Hombre (30-60): }(11,6 \times M C)+879 \\
\text { Hombre (>60): }(13,5 \times M C)+487\end{array}$ & Sana \\
\hline $\begin{array}{l}\text { Owen y colaboradores } \\
\text { (1988) (20) }\end{array}$ & $\begin{array}{l}\mathrm{n}=44 \text { mujeres (incluyó } 8 \text { atletas), } \\
\text { sin especificación racial/étnica, 18- } \\
65 \text { años, 43-143 kg. }\end{array}$ & $\begin{array}{l}\text { Mujer: } 795+(7,18 \times M C) \\
\text { Hombre: } 879+10,2 \times M C\end{array}$ & Sana \\
\hline $\begin{array}{l}\text { Mifflin y colaboradores } \\
(1990)(22)\end{array}$ & $\begin{array}{l}\mathrm{n}=498,19-78 \text { años }(44 \pm 14) .251 \\
\text { hombres }(87,5 \pm 14,4 \mathrm{~kg}) .247 \mathrm{mu}- \\
\text { jeres }(70,2 \pm 14,1 \mathrm{~kg}) .\end{array}$ & $\begin{array}{l}\text { Mujer: }(10 \times M C)+(6,25 \times A)-(4,92 \times \\
\text { edad })-161 \\
\text { Hombres: }(10 \times M C)+(6,25 \times A)-(4,92 \times \\
\text { edad })+5\end{array}$ & Sana \\
\hline $\begin{array}{l}\text { Gougeon y } \\
\text { colaboradores (2002) (30) }\end{array}$ & $\begin{array}{l}65 \text { adultos, } 40 \text { mujeres y } 25 \\
\text { hombres, IMC promedio } 37 \mathrm{~kg} / \mathrm{m}^{2} \text {. }\end{array}$ & $375+(85 \times M C)-(48 \times M G)+(63 \times G A)$ & Diabetes mellitus \\
\hline $\begin{array}{l}\text { Huang y colaboradores } \\
(2004)(31)\end{array}$ & $\begin{array}{l}1088 \text { adultos, edad media } 44,9+/- \\
12,7 \text { años con IMC }>35 \mathrm{~kg} / \mathrm{m}^{2}, 142 \\
\text { con diabetes mellitus tipo } 2 \text {. }\end{array}$ & $\begin{array}{l}71,767-(2,337 \times \text { edad })+(257,293 \times 0)+ \\
(9,996 \times M C)+(4,132 \times A)+(145,959 \times 1)\end{array}$ & $\begin{array}{l}\text { Diabetes mellitus/ } \\
\text { Obesidad }\end{array}$ \\
\hline $\begin{array}{l}\text { Rodrigues y } \\
\text { colaboradores (2010) (32) }\end{array}$ & 760 mujeres de 18 a 65 años & $\begin{array}{l}\mathrm{IMC}>35 \mathrm{~kg} / \mathrm{m}^{2}: 172,19+(10,93 \times \mathrm{MC})+ \\
(3,10 \times \mathrm{A})-(2,55 \times \text { edad }) \\
\mathrm{IMC}<35 \mathrm{~kg} / \mathrm{m}^{2}: 407,57+(9,58 \times \mathrm{MC})+(2,05 \\
\times A)-(1,74 \times \text { edad })\end{array}$ & Sana \\
\hline Harrington (1997) (23) & $\begin{array}{l}\mathrm{n}=498,19-78 \text { años }(44 \pm 14) .251 \\
\text { hombres }(87,5 \pm 14,4 \mathrm{~kg}) .247 \\
\text { mujeres }(70,2 \pm 14,1 \mathrm{~kg}) .\end{array}$ & $\begin{array}{l}\text { Mujer: }(\text { IMC x 28,15) - (edad x 6,44) + } 905 \text {. } \\
\text { Hombre: }(\text { IMC x 28,15) - (edad x 6,44) + } \\
1290 \text {. }\end{array}$ & Obesidad \\
\hline Oxford (2005) (28) & $\begin{array}{l}\mathrm{n}=10522 \text { (5794 hombres y } 4702 \\
\text { mujeres) }\end{array}$ & 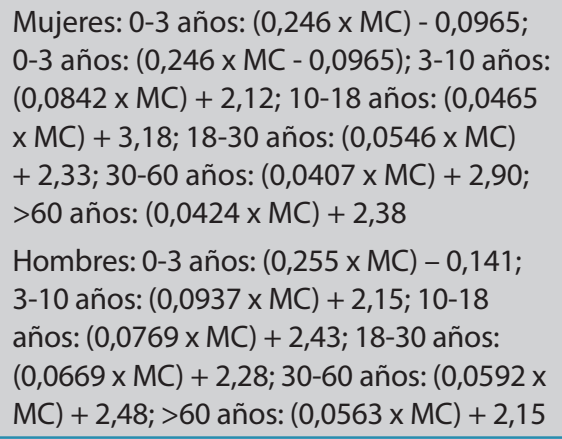 & Sanos \\
\hline
\end{tabular}

A: altura (cm); GA: glucosa en ayunas (mM); H: humedad; IMC: índice de masa corporal (kg/m2); MC: masa corporal (kg); MG: masa grasa; SC: superficie corporal; T: temperatura. 
duos con baja TMB $(44,45)$. Estas afirmaciones se observaron previamente en otros estudios, aunque con grupos poblacionales más específicos y con menor número de sujetos (46-48).

\section{Tasa metabólica en enfermedades crónicas no transmisibles (ECNT)}

El cuerpo humano, en su búsqueda por mantener un estado de equilibrio energético, en presencia de alteraciones que afecten esta homeostasis, como la restricción calórica aguda y las enfermedades crónicas, puede llevar a una disminución de la TMB, la cual está sujeta a variaciones al ser influenciada por diferentes factores como la composición corporal y los cambios hormonales, presentes en la mayoría de las $\operatorname{ECNT}(49,50)$. Como se mencionó, a pesar de que la utilización de ecuaciones predictivas es la forma más sencilla para calcular la TMB se ha observado, particularmente en sujetos con condiciones adicionales, una sobre o subestimación de los resultados, las cuales pueden asociarse con las diferencias en las características de la población evaluada en comparación con la población participante en los estudios de las cuales derivan originalmente estas ecuaciones (51). Christensen y colaboradores midieron la TMB por CI y la calcularon con la ecuación predictiva MSJ, al encontrar los niveles mayores en aquellos sujetos con tensión arterial elevada $(p<0,05)$ y levemente menores en mujeres con LDL elevada, a su vez, luego de ser evaluados por aproximadamente $13,6+/-12,8$ meses y de obtener una pérdida de peso promedio de $8+/-11 \mathrm{~kg}$ y 5,5 +/- 7,3 kg para hombres y mujeres, respectivamente (22). Se encontró que no hubo una disminución significativa de la TMB, independiente del género o del estado de riesgo de salud de la condición crónica $(p>0,05)(52)$. De las ecuaciones predictivas existentes, la de Harris y MSJ son las dos más utilizadas en condiciones clínicas, estas son específicas según el género y usan la edad, altura y peso para predecir la TMB (53)y. Sin embargo, al no tener en cuenta los efectos de las comorbilidades asociadas a obesidad como la tensión arterial y los valores de LDL, no son exactas dado el efecto que estos han mostrado tener efecto sobre la TMB.

En mujeres con diabetes mellitus tipo 2, con una media de hemoglobina glucosilada de 7,7 \%, Figueiredo y colaboradores compararon la medición de la TMB por CI vs. la estimada por 7 ecuaciones predictivas, que incluyen la de HarrisBenedict, FAO/WHO/UNU, Owen, Mifflin, Gougeon, Huang y Rodrigues, encontrando sobreestimación de las medidas con Huang (11,26 \%, 4-18), FAO/WHO/UNU (10,58 \%; 3-18) y subestimación con Mifflin (-2,58 \%; [-8] - 3), siendo la medida más cercana la de Owen y Gougeon $(32,50)$. A pesar no haber una evidencia clara que indique como la diabetes influencia el metabolismo basal, se ha observado niveles mayores de TMB en estos pacientes al compararlos con sujetos control sin la enfermedad (9). Se ha planteado como posible relación el aumento del recambio proteico, los altos niveles de ácidos grasos libres en plasma en ayunas, el aumento de la oxidación de carbohidratos, la hiperactividad simpática y el incremento de la gluconeogénesis, los cuales se asocian con mayor consumo energético $(52,54-56)$.

En pacientes diabéticos tipo 2 con neuropatía periférica, la TMB ha mostrado correlacionarse positivamente con el HOMAIR, grasa visceral, glucosa basal y masa musculoesquelética (57). En el caso de pacientes con falla renal y desnutrición, se ha observado disminución de la TMB. La neumopatía crónica y el cáncer pulmonar se asocia con incremento en TMB (52).

\section{Discusión}

Con los cambios de estilo de vida modernos se ha fomentado la ingesta de comidas hipercalóricas y el sedentarismo, lo cual genera una alteración entre el aporte y el gasto energético; este disbalance favorece el incremento de la reserva energética con hipertrofia e hiperplasia del tejido adiposo, lo que favorece el aumento progresivo de la inflamación y promueve así el desarrollo de patologías como el sobrepeso y la obesidad, y sus comorbilidades, como la diabetes, la hipertensión, el cáncer y las enfermedades cardio y cerebrovasculares.

El GET depende en gran medida de la TMB, cuyo valor representa hasta el $75 \%$ de la energía utilizada por el cuerpo en condiciones de reposo físico y mental. Aunque idealmente debe ser medida por métodos como la calorimetría indirecta, a lo largo de los años, dada su baja disponibilidad, se han desarrollado ecuaciones predictivas basadas en variables que incluyen la edad, género y composición corporal. En la revisión realizada, se observó una correlación subóptima de estas ecuaciones con los métodos estándar, mostrando una correlación del 70 \% en el mejor de los casos. Lo anterior se podría explicar por las poblaciones usadas para los estudios presentan limitaciones o tienen características específicas que no permiten su aplicabilidad a la población general, con lo cual en su mayoría se sobreestima la TMB y los requerimientos energéticos totales (58).

Aunque tradicionalmente se ha descrito que la masa libre de grasa es el principal condicionante de la TMB y, por tanto, del GET, en el caso de la obesidad, considerada como un exceso disfuncional de tejido graso, los cambios de los niveles de grasa subcutánea y visceral son de gran relevancia como componente clave del GET, lo cual no se tiene discriminado en las ecuaciones mencionadas en este artículo y limita su utilización, lo que lleva al uso de herramientas, como la calorimetría indirecta, la cual no es siempre asequible y tiene baja disponibilidad, por lo cual no es práctico establecer su medición de forma rutinaria en el seguimiento clínico de un paciente.

Por lo anterior podemos concluir la importancia de considerar realizar estudios que permitan esclarecer la correlación de los diferentes compartimentos grasos en composición corporal, como lo son la grasa visceral, la masa grasa total o segmentaria, el porcentaje graso y los índices de riesgo antropométrico (índice cintura/altura, índice cintura/cadera, entre 


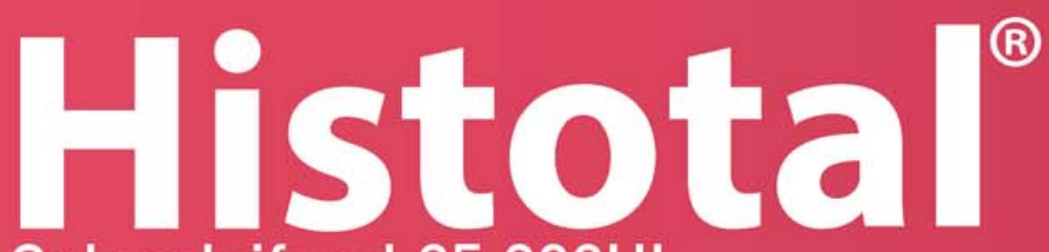

Colecalciferol 25.000UI

\section{Primera terapia oral de altas dosis de Vitamina D en Colombia}

En una base de aceite de oliva con presentación en ampolla de plástico de $1 \mathrm{ml}$ con 25,000 UI de Vitamina D

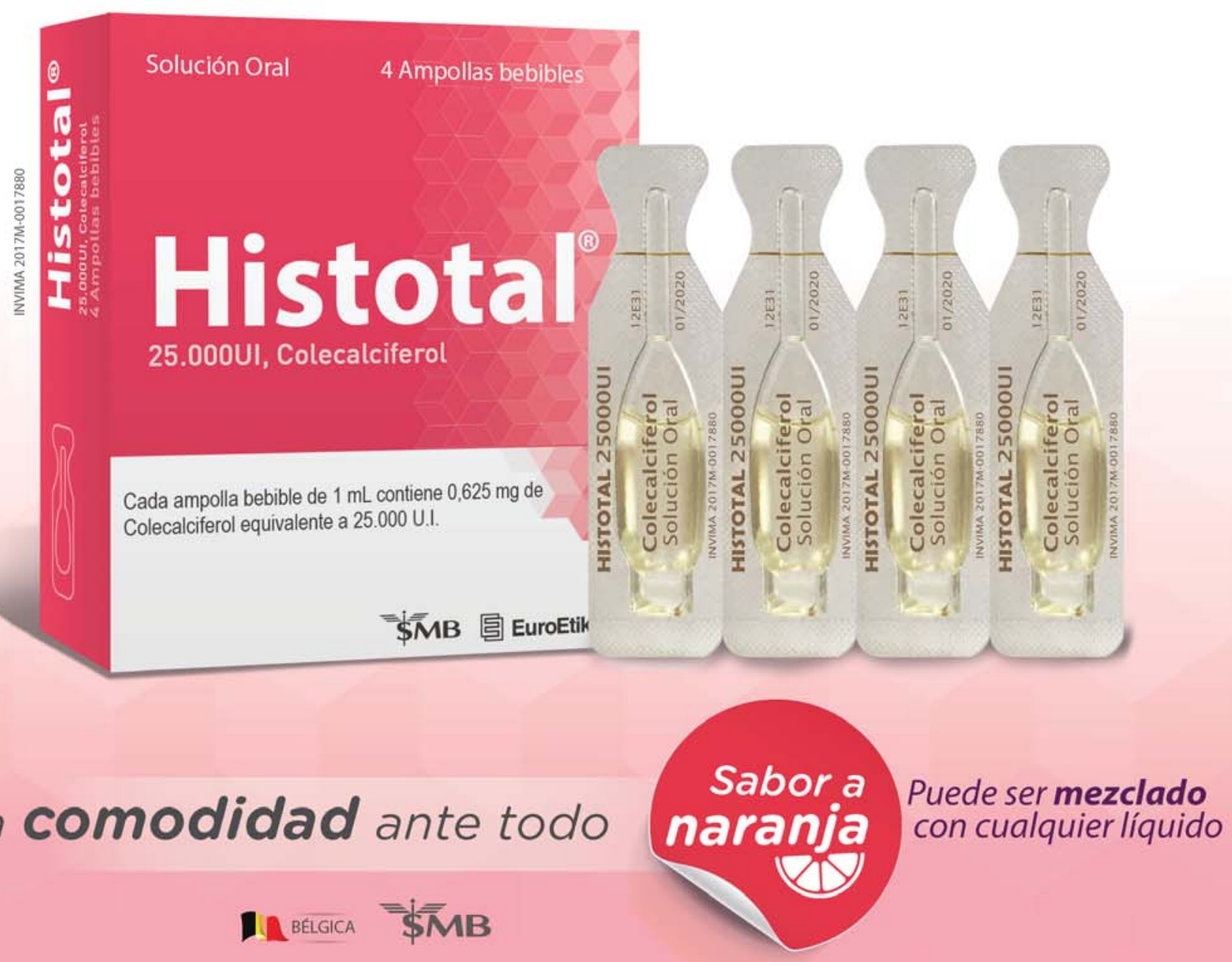


otros) para poder determinar la necesidad de crear fórmulas que puedan tener mayor impacto basadas en el componente graso corporal para sujetos con obesidad. Esta última se define como un estado patológico que se caracteriza por un exceso o acumulación excesiva y general de la grasa en el cuerpo, por lo que, por su definición, parece lógico considerar nuevos métodos que calculen la TMB en pacientes con gran cúmulo de grasa, que sean de fácil uso en el día a día del médico dedicado a ver este tipo de enfermedades, con el fin de evaluar el efecto de condiciones, como los cambios en la alimentación y el ejercicio, y la predicción en la pérdida o aumento de peso.
De cierta forma, la más adecuada para considerar su uso en paciente según la edad y peso sería la ecuación de Oxford, la cual, a su vez, es poco popular. Por ello, es necesario realizar estudios en esta población, en particular, que confirmen qué variables deben tenerse en cuenta en la predicción de la TMB.

\section{Conflicto de interés}

Ninguno.

\section{Fuentes de financiamiento}

\author{
Ninguna.
}

\section{Referencias}

1. Chooi YC, Ding C, Magkos F. The epidemiology of obesity. Metabolism [Internet]. 2019;92:6-10.

2. Seravalle G, Grassi G. Obesity and hypertension. Pharmacol Res. 2017;122:1-7.

3. Klop B, Elte JWF, Cabezas MC. Dyslipidemia in Obesity: Mechanisms and Potential Targets. Nutrients. 2013;5(4):1218-40.

4. Boles A, Kandimalla R, Reddy PH. Dynamics of diabetes and obesity: Epidemiological perspective. Biochim Biophys Acta - Mol Basis Dis [Internet] 2017;1863(5):1026-36.

5. Ortega-Loubon C, Fernández-Molina M, Singh G, Correa R. Obesity and its cardiovascular effects. Diabetes Metab Res Rev. 2019;35(4).

6. Francisco V, Pérez T, Pino J, López V, Franco E, Alonso A, et al. Biomechanics, obesity, and osteoarthritis. The role of adipokines: When the levee breaks. J Orthop Res. 2018;36(2):594-604.

7. Ackerman SE, Blackburn OA, Marchildon F, Cohen P. Insights into the Link Between Obesity and Cancer. Curr Obes Rep. 2017;6(2):195-203.

8. Gouma PI, Alkhader M, Stanaćević M. Metabolic rate monitoring and weight reduction/management. 2014 36th Annu Int Conf IEEE Eng Med Biol Soc EMBC 2014. 2014;3184-7.

9. Doros R, Delcea A, Mardar L, Petcu L. Basal metabolic rate in metabolic disorders. Medicine (Baltimore). 2015;17(2):137-43.

10. Pavlidou E, Petridis D, Tolia M, Tsoukalas N, Poultsidi A, Fasoulas A, et al. Estimating the agreement between the metabolic rate calculated from prediction equations and from a portable indirect calorimetry device: An effort to develop a new equation for predicting resting metabolic rate. Nutr Metab. 2018;15(1):1-9.

11. Weyer C, Snitker S, Rising R, Bogardus C, Ravussin E. Determinants of energy expenditure and fuel utilization in man: Effects of body composition, age, sex, ethnicity and glucose tolerance in 916 subjects. Int J Obes. 1999;23(7):715-22.

12. Gropper S SJ. Advanced nutrition and human metabolism. Biochemical Education. 2013

13. Ainsworth BE, Haskell WL, Leon AS, Jacobs DR, Montoye HJ, Sallis JF, et al Compendium of physical activities: classification of energy costs of human physical activities. Med Sci Sports Exerc. 1993;25(1):71-80.

14. Wang Z, Heshka S, Gallagher D, Boozer CN, Kotler DP, Heymsfield SB. Resting energy expenditure-fat-free mass relationship: new insights provided by body composition modeling. Am J Physiol Metab. 2017;279(3):E539-45.

15. Lazzer S, Bedogni G, Lafortuna CL, Marazzi N, Busti C, Galli R, et al. Relationship between basal metabolic rate, gender, age, and body composition in 8,780 white obese subjects. Obesity. 2010;18(1):71-8.

16. Choe SS, Huh JY, Hwang IJ, Kim JI, Kim JB. Adipose tissue remodeling: Its role in energy metabolism and metabolic disorders. Front Endocrinol (Lausanne). 2016;7(APR):1-16

17. Harris JA, Benedict FG. A Biometric Study of Human Basal Metabolism. Proc Natl Acad Sci U S A. 1918;4(12):370-3.

18. Roza AM, Shizgal HM. The Harris Benedict equation reevaluated: Resting energy requirements and the body cell mass. Am J Clin Nutr. 1984;40(1):168-82.

19. Schofield WN. Predicting basal metabolic rate, new standards and review of previous work. Hum Nutr Clin Nutr. 1985;39(1):5-41.

20. Owen OE. Resting Metabolic Requirements of Men and Women. Mayo Clinic Proceedings. 1988;63(5):503-10.
21. Livesey G. Energy and protein requirements the 1985 report of the 1981 Joint FAO/WHO/UNU Expert Consultation. Nutr Bull. 1987.

22. Mifflin MD, St Jeor ST, Hill LA, Scott BJ, Daugherty SA, Koh YO. A new predictive equation for resting energy expenditure in healthy individuals. Am J Clin Nutr. 1990;51(2):241-7.

23. Harrington ME., St. Jeor ST. SL. Predicting resting energy expenditure from body mass index: practical applications and limitations: annual conference proceedings, North American Association for the Study of Obesity. J Obes Res. 1997;5.

24. Quenouille MH, Boyne AW, Fisher WB LI. Statistical Studies of Recorded Energy Expenditure of Man. Basal Metabolism Related to Sex, Stature, Age, Climate, and Race. Commonw Bur Anim Nutr. 1951;17.

25. Henry CJ, Rees DG. New predictive equations for the estimation of basa metabolic rate in tropical peoples. Eur J Clin Nutr. 1991;45(4):177-85.

26. Piers LS, Diffey B, Soares MJ, Frandsen SL, McCormack LM, Lutschini MJ, et al. The validity of predicting the basal metabolic rate of young Australian men and women. Eur I Clin Nutr. 1997;51(5):333-7.

27. Wahrlich V, Anjos LA. Validação de equações de predição da taxa metabólica basal em mulheres residentes em Porto Alegre, RS, Brasil. Rev Saude Publica. 2001:35(1):39-45.

28. Henry CJK. Basal metabolic rate studies in humans : measurement and development of new equations. Public Health Nutr. 2005;8:1133-52.

29. Frankenfield DC, Muth ER, Rowe WA. The Harris-Benedict studies of human basal metabolism: History and limitations. J Am Diet Assoc. 1998;98(4):439-45.

30. Gougeon R, Lamarche M, Yale JF, Venuta T. The prediction of resting energy expenditure in type 2 diabetes mellitus is improved by factoring for glycemia. Int J Obes. 2002;26(12):1547-52.

31. Huang KC, Kormas N, Steinbeck K, Loughnan G, Caterson ID. Resting metabolic rate in severely obese diabetic and nondiabetic subjects. Obes Res. 2004;12(5):840-5

32. Rodrigues AE, Mancini MC, Dalcanale L, Melo ME de, Cercato C, Halpern A. Characterization of metabolic resting rate and proposal of a new equation for a female Brazilian population. Arq Bras Endocrinol Metabol. 2010;54(5):470-6

33. Achamrah N, Jésus P, Grigioni S, Rimbert A, Petit A, Déchelotte P, et al Validity of predictive equations for resting energy expenditure developed for obese patients: Impact of body composition method. Nutrients. 2018;10(1):1-11.

34. Dulloo AG, Jacquet J, Solinas G, Montani JP, Schutz Y. Body composition phenotypes in pathways to obesity and the metabolic syndrome. Int J Obes. 2010;34(2):S4-17.

35. Do R, Bailey SD, Desbiens K, Belisle A, Montpetit A, Bouchard C, et al. Genetic variants of FTO influence adiposity, insulin sensitivity, leptin levels, and resting metabolic rate in the Quebec family study. Diabetes. 2008;57(4):1147-50

36. Loos RJF, Ruchat S, Rankinen T, Tremblay A, Pérusse L, Bouchard C. Adiponectin and adiponectin receptor gene variants in relation to resting metabolic rate, respiratory quotient, and adiposity-related phenotypes in the Quebec Family Study. Am J Clin Nutr. 2007;85(1):26-34.

37. Lara-Castro C, Luo N, Wallace P, Klein RL, Garvey WT. Adiponectin multimeric complexes and the metabolic syndrome trait cluster. Diabetes. 2006; 
38. Moradi S, Mirzaei K, Abdurahman AA, Keshavarz SA, Hossein-nezhad A. Mediatory effect of circulating vaspin on resting metabolic rate in obese individuals. Eur J Nutr. 2016;

39. Ansar H, Mirzaei K, Malek A, Najmafshar A, Hossein-Nezhad A. Possible resting metabolic rate modification by the circulating RBP4 in obese subjects. Diabetes Metab Syndr Clin Res Rev. 2015;

40. Yang R-Z, Lee M-J, Hu H, Pray J, Wu H-B, Hansen BC, et al. Identification of omentin as a novel depot-specific adipokine in human adipose tissue: possible role in modulating insulin action. Am J Physiol Metab. 2006;55(1):249-59.

41. Farnaz Sepandar, Elaheh Rashidbeydi, Zhila Maghnooli, Leila KhorramiNezhad, Massomeh Hajizadehoghaz KM. The association between resting metabolic rate and metabolic syndrome may be mediated by adipokines in overweight and obese women. Diabetes Metab Syndr Clin Res Rev. 2019;13:530-4.

42. Katie R. Hirsch, Abbie E. Smith-Ryan, Malia N.M. Blue, Meredith G. Mock and ETT. Influence of Segmental Body Composition and Adiposity Hormones on Resting Metabolic Rate and Substrate Utilization in Overweight and Obese Adults. J Endocrinol Invest. 2017;40(6):635-43.

43. Sabounchi NS, Rahmandad H, Ammerman A. Best-fitting prediction equations for basal metabolic rate: Informing obesity interventions in diverse populations. Int J Obes. 2013;37(10):1364-70.

44. Wang Z, Ying Z, Bosy-Westphal A, Zhang J, Heller M, Later W, et al. Evaluation of specific metabolic rates of major organs and tissues: Comparison between nonobese and obese women. Am J Hum Biol. 2011;23(3):333-8.

45. Anthanont $P$, Jensen MD. Does basal metabolic rate predict weight gain? Am J Clin Nutr. 2016;104(4):959-63.

46. Tataranni PA, Harper IT, Snitker S, Del Parigi A, Vozarova B, Bunt J, et al Body weight gain in free-living Pima Indians: Effect of energy intake vs expenditue. Int J Obes. 2003;

47. Ravussin E, Lillioja S, Knowler WC, Christin L, Freymond D, Abbott WGH, et al. Reduced Rate of Energy Expenditure as a Risk Factor for Body-Weight Gain. N Engl J Med. 1988;318(8):467-72.
48. Buscemi S, Verga S, Caimi G, Cerasola G. Low relative resting metabolic rate and body weight gain in adult Caucasian Italians. Int J Obes. 2005;29(3):287-91.

49. Bray G. Effect of caloric restriction on energy expenditure in obese patients. Lancet. 1969;2(7617):397-8

50. de Figueiredo Ferreira M, Detrano F, Coelho GM de O, Barros ME, Serrão Lanzillotti R, Firmino Nogueira Neto J, et al. Body Composition and Basa Metabolic Rate in Women with Type 2 Diabetes Mellitus. J Nutr Metab. 2014;1-9.

51. Kien CL, Ugrasbul F. Prediction of daily energy expenditure during a feeding trial using measurements of resting energy expenditure, fat-free mass, or Harris-Benedict equations. Am J Clin Nutr. 2004;80(4):876-80.

52. Christensen RAG, Raiber L, Wharton S, Rotondi MA, Kuk JL. The associations of resting metabolic rate with chronic conditions and weight loss. Clin Obes. 2017;7(2):70-6.

53. Miller S, Milliron BJ, Woolf K. Common prediction equations overestimate measured resting metabolic rate in young hispanic women. Topics in Clinical Nutrition. 2013;28(2):120-35.

54. Payne PR, Waterlow JC. Relative energy requirements for maintenance, growth, and physical activity. The Lancet. 1971;2(7717):210-1.

55. Tredget EE, Yu YM. The metabolic effects of thermal injury. World J Surg 1992;16(1):68-79.

56. Consoli A, Nurjhan N, Capani F, Gerich J. Predominant role of gluconeogenesis in increased hepatic glucose production in NIDDM. Diabetes. 1989;38(5):550-7

57. Sampath Kumar A, Arun Maiya G, Shastry BA, Vaishali K, Maiya S, Umakanth $\mathrm{S}$. Correlation between basal metabolic rate, visceral fat and insulin resistance among type 2 diabetes mellitus with peripheral neuropathy. Diabetes Metab Syndr Clin Res Rev. 2019;13(1):344-8.

58. De Oliveira EP, Orsatti FL, Teixeira O, Maestá N, Burini RC. Comparison of predictive equations for resting energy expenditure in overweight and obese adults. J Obes. 2011;534714 